\title{
Electron collisions with CO: Elastic and vibrational excitation cross sections
}

\author{
M. Allan \\ Department of Chemistry, University of Fribourg, chemin du Musée 9, CH-1700 Fribourg, Switzerland
}

\begin{abstract}
Absolute differential elastic and vibrational excitation cross sections up to $v=11$ were measured for $\mathrm{CO}$ in scattering angle ranges extending to $180^{\circ}$ at energies between 0.2 and $5 \mathrm{eV}$ (and an elastic measurement at $10 \mathrm{eV}$ ). The lowest angles were $0^{\circ}$ for inelastic scattering and between $5^{\circ}$ and $20^{\circ}$ for elastic scattering, depending on energy. Integral cross sections were derived by integrating under the angular distributions and compared with previous beam and swarm measurements. The sum of the integral cross sections agrees very well with the available transmission measurements of the grand total cross section, thus validating the present measurements. The present elastic differential and integral cross sections are in excellent agreement with the best available measurement [Gibson et al., J. Phys. B 29, 3197 (1996)], but the $v=1$ inelastic cross section is about $25 \%$ higher. This could have consequences for simulations of cometary and planetary atmospheres.
\end{abstract}

\section{INTRODUCTION}

Simulations of the upper atmospheres of Venus and Mars and cometary comae [1-3] require accurate values of cross sections for vibrational excitation of carbon monoxide by low-energy electron impact. Although electron collisions with $\mathrm{CO}$ have been studied many times, there are only few truly quantitative measurements which encompass wide angular ranges and thus yield absolute angle-integrated cross sections (ICS). Furthermore, there is not always full agreement about the cross section magnitudes. The present work aims at improving the existing data by measuring the differential cross sections (DCS) over the entire angular range and thus permitting the determination of assumption-free integral cross sections and by making an elaborate effort to determine and take into account the spectrometer response functions to improve the accuracy of the vibrationally inelastic cross sections.

Early work on vibrational excitation of $\mathrm{CO}$ was covered in the 1973 review by Schulz [4]. The review of Brunger and Buckman [5] covers the work up to 2002. Integral cross sections were reviewed by Zecca et al. [6]. Early work on vibrational excitation up to $v=8$ was that of Schulz [7], performed at a fixed angle of $72^{\circ}$. He estimated the absolute integral values by reference to the total cross section under the assumption of isotropic scattering. Differential cross sections for elastic scattering and for the excitation of vibrational levels up to $v=7$ (their angular distributions in the range $5^{\circ}-110^{\circ}$, yielding an improved integral cross section) and integral cross sections were measured by Ehrhardt et al. [8]. Tanaka et al. [9] measured the elastic cross sections in the range $15^{\circ}-130^{\circ}$, later used by Chutjian and Tanaka to derive the absolute inelastic cross sections [10]. Jung et al. [11] performed a high-resolution $(\sim 10 \mathrm{meV})$ experiment and obtained absolute differential cross sections for pure rotational and for ro-vibrational $(v=1)$ excitation. Sums over all rotational transitions can be compared to the present data. Sohn et al. [12] measured the elastic and $v=1$ cross sections in the energy range below the ${ }^{2} \Pi$ resonance, from threshold to $1.5 \mathrm{eV}$. The most recent and authoritative study, of the elastic and $v=1$ cross sections, is that Gibson et al. [13]. A high-resolution (10-12 meV) study of rotational excitation was reported by Gote and Ehrhardt [14], at energies of 5-200 eV. Relative cross sections for excitation of vibrational states up to $v=15$ were measured with a magnetically collimated trochoidal spectrometer by Allan [15]. Poparić et al. [16] studied vibrational excitation with a crossed-beam double trochoidal electron spectrometer. They then used their earlier measured information that the forward-to-backward inelastic cross section ratio is $1.00 \pm 0.06$ [17] and the absolute value of Gibson et al. [13] to derive absolute integral cross sections.

Momentum-transfer and inelastic-collision cross sections were calculated from measured values of the electron drift velocity by Hake and Phelps [18] and later by Land [19]. Grand total cross sections are also relevant to the present work in the sense that they can be compared to the sum of the present partial (elastic + vibrational excitation up to $v=11$ ) integral cross sections. Grand total cross sections particularly suitable for comparison with the present work are those of Ramsauer and Kollath [20], Buckman and Lohmann [21], Kwan et al. [22], Szmytkowski et al. [23], as well as the selection of "recommended" data of Kanik et al. [24].

Theory of angular distributions for $\mathrm{CO}$ was presented by Read [25]. $R$-matrix calculations were performed by Morgan [26] and Morgan and Tennyson [27].

\section{EXPERIMENT}

The measurements were performed using a spectrometer with hemispherical analyzers [28-30]. The energy resolution was about $14 \mathrm{meV}$ in the energy-loss mode, corresponding to about $10 \mathrm{meV}$ in the incident electron beam, at a beam current of around $200 \mathrm{pA}$. The energy of the incident beam was calibrated on the $19.365 \mathrm{eV} \mathrm{[31]}{ }^{2} S$ resonance in helium and is accurate to within $\pm 10 \mathrm{meV}$. The instrumental response function was determined on elastic scattering in helium and all spectra were corrected as described earlier [29].

Absolute values of the elastic cross sections were determined by the relative flow technique as described by Nickel et al. [32] using the theoretical helium elastic cross sections of Nesbet [33] as a reference. The confidence limit is about $\pm 15 \%$ for the elastic cross sections and $\pm 20 \%$ for the inelastic cross sections (two standard deviations). The $\mathrm{CO}$ and helium 


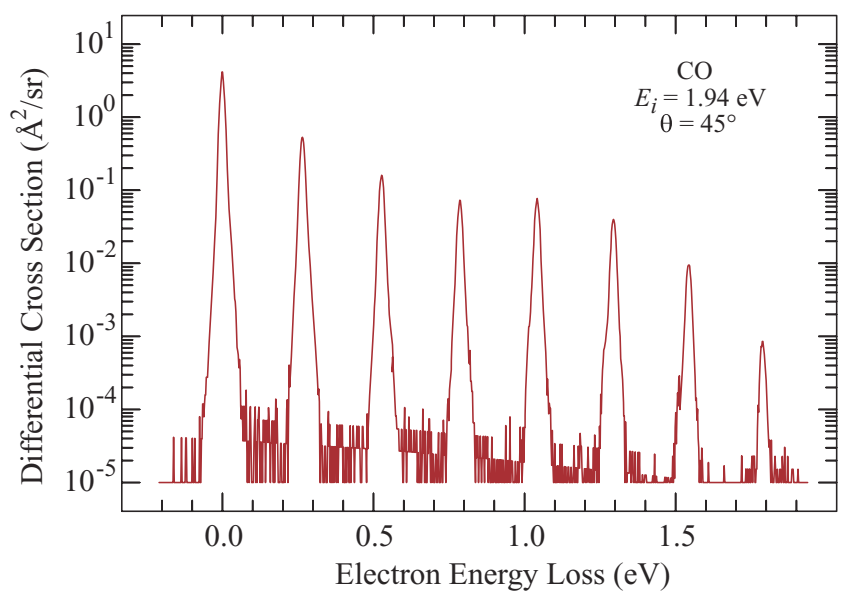

FIG. 1. (Color online) Typical electron energy loss spectrum used to determine the inelastic-to-elastic cross-section ratios. The spectrum is corrected for the instrumental response function. No background was subtracted.

pressures in the gas inlet line during the absolute measurements were kept low, typically 0.1 and 0.2 mbars, respectively. Background was determined by recording signal with gas flowing into the main chamber via a bypass line and not the nozzle. This background was generally negligible except in the more forward scattering and at low energies - but for consistency the "bypass signal" was subtracted for the absolute measurements even when it was very low. The excitation functions and the angular distributions were measured at higher pressures and background was subtracted only when it was significant. Absolute inelastic cross sections were derived by integrating the areas under the elastic and inelastic peaks in energy-loss spectra recorded at constant incident energies, corrected for instrumental response functions, such as that shown in Fig. 1.

The angular distributions were measured using combined mechanical setting of the analyzer and magnetic deflection with the magnetic angle changer [34,35], correcting the curves for the instrumental response function (determined on helium and, for angles near $0^{\circ}$, on the $v=1$ excitation cross section in $\mathrm{N}_{2}$ [29,36]), and fitting them to the discrete absolute values measured at $45^{\circ}, 90^{\circ}, 135^{\circ}$, and $180^{\circ}$. Details of the procedure were described in Ref. [29]. The magnetic deflection was incremented in steps of $2.5^{\circ}$.

Rotational excitation is, particularly in the resonance region, strong [11] and leads to a substantial broadening of the energy-loss bands. Consequently, care had to be taken to derive all cross sections from the areas under the energy-loss bands and not from their heights. For angular distributions, and for the absolute measurements, an energy-loss peak was recorded at each energy and angle, and was then numerically integrated. Two methods are used for the excitation functions. One consists of recording a number of excitation functions at energy losses around the nominal energy loss of the process being recorded (elastic or a given vibrational level), thus covering the entire width of rotational excitations, and then making the sum. The other consists of recording a number of energy-loss spectra in the energy range of interest, then deriving numerically their heights and areas and constructing a "height-to-area correction function," used to correct an excitation function recorded at the top of the energy-loss peak. Both methods gave consistent results.

\section{RESULTS AND DISCUSSION}

\section{A. Differential cross sections}

Figures 2-7 show the elastic and vibrational excitation cross sections as a function of electron energy at selected angles. The elastic cross sections cannot be recorded in the forward direction, but selected vibrational excitation cross sections recorded at $0^{\circ}$ are shown in Fig. 8. Cross sections for some of the higher vibrational channels are shown in the supplementary material [37], together with all data in digital form. Absolute cross sections could not be measured directly at $0^{\circ}$ because the elastic signal cannot be recorded. The excitation functions were therefore normalized to the values taken from the angular distributions, Figs. 9-11. This normalization is less reliable than that used at the higher scattering angles. The data

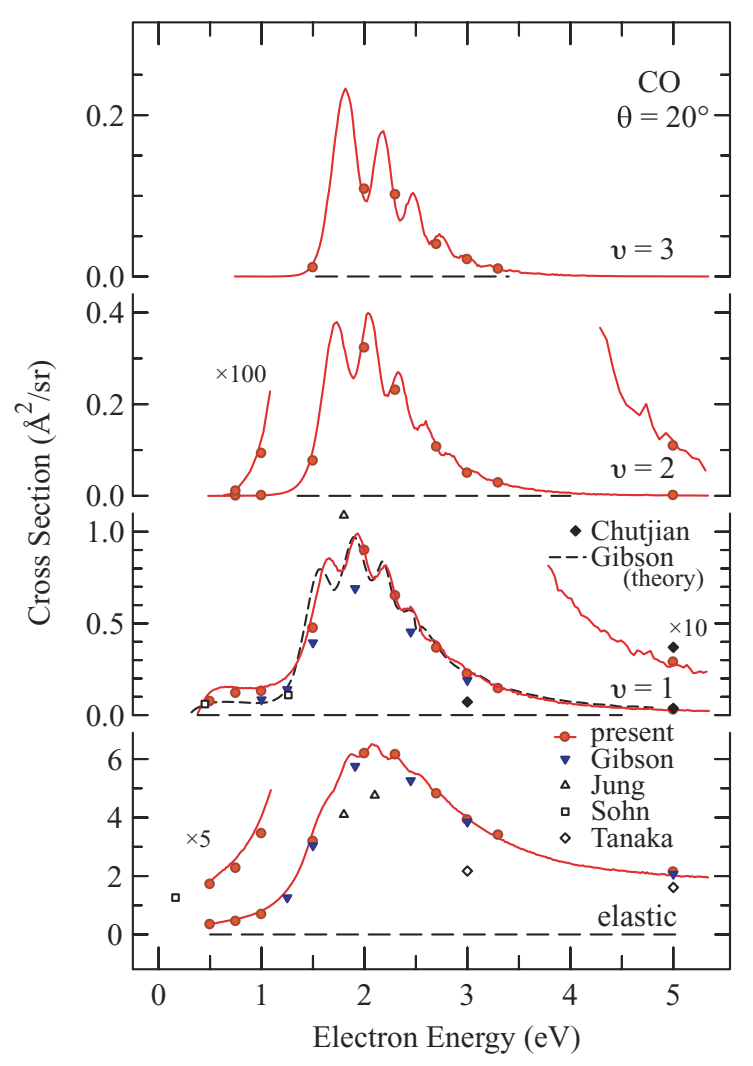

FIG. 2. (Color online) Cross sections at $20^{\circ}$, shown as a function of electron energy. Red (continuous) lines show the excitation functions. Red circles show the results of individual absolute measurements. The elastic and the $v=1$ excitation functions were normalized to the absolute measurements at $2 \mathrm{eV}$. The normalization of the $v=2$ and $v=3$ excitation functions is, via the relative signal intensities, also to the $v=1$ absolute value at $2 \mathrm{eV}$. The remaining red dots were thus not used for normalization and serve only as a check of the internal consistency of the data sets. The data of Gibson et al. [13], Jung et al. [11] (interpolated between their $15^{\circ}$ and $30^{\circ}$ data), Sohn et al. [12], Tanaka et al. (elastic) [9] and Chutjian and Tanaka $(v=1)[10]$ are shown for comparison. 


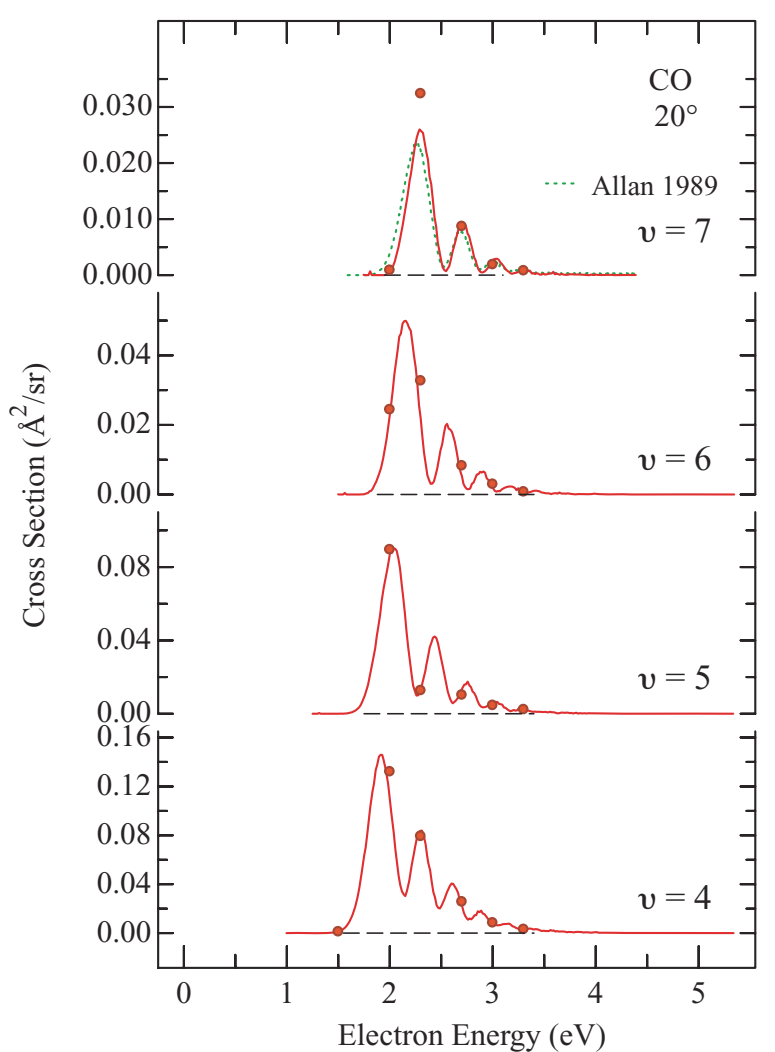

FIG. 3. (Color online) Continuation from Fig. 2. The $v=7$ data from Ref. [15], normalized to the present data at the peak of the $20^{\circ}$, $v=1$ spectrum, is shown for comparison.

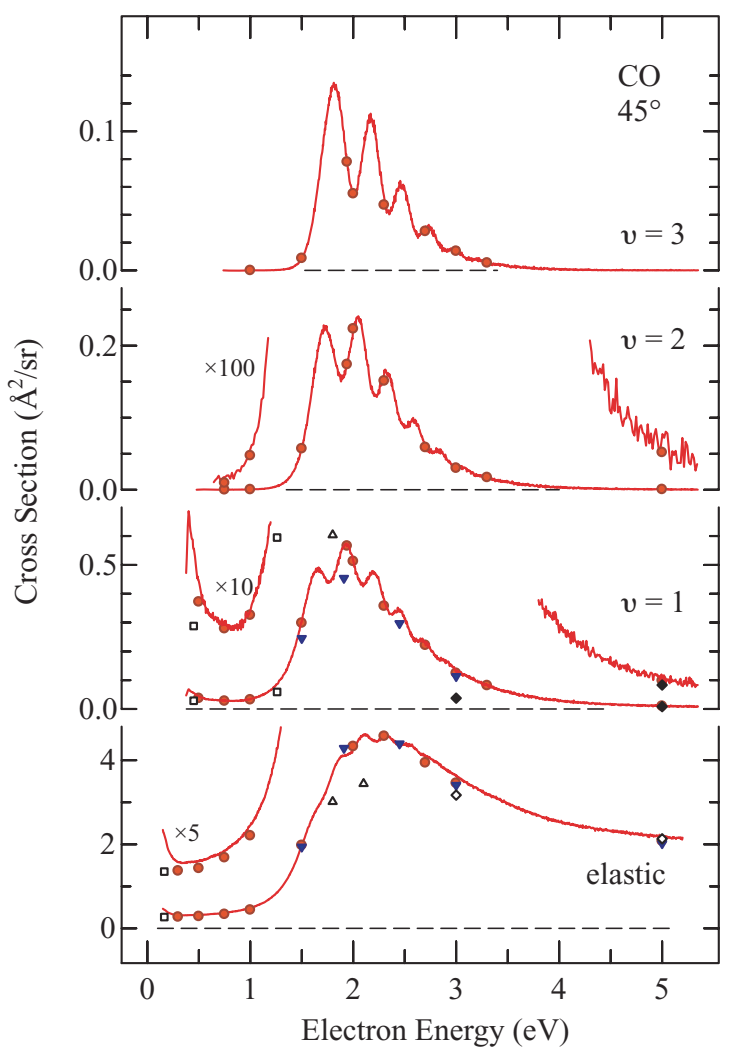

FIG. 4. (Color online) As Fig. 2, but at $45^{\circ}$.

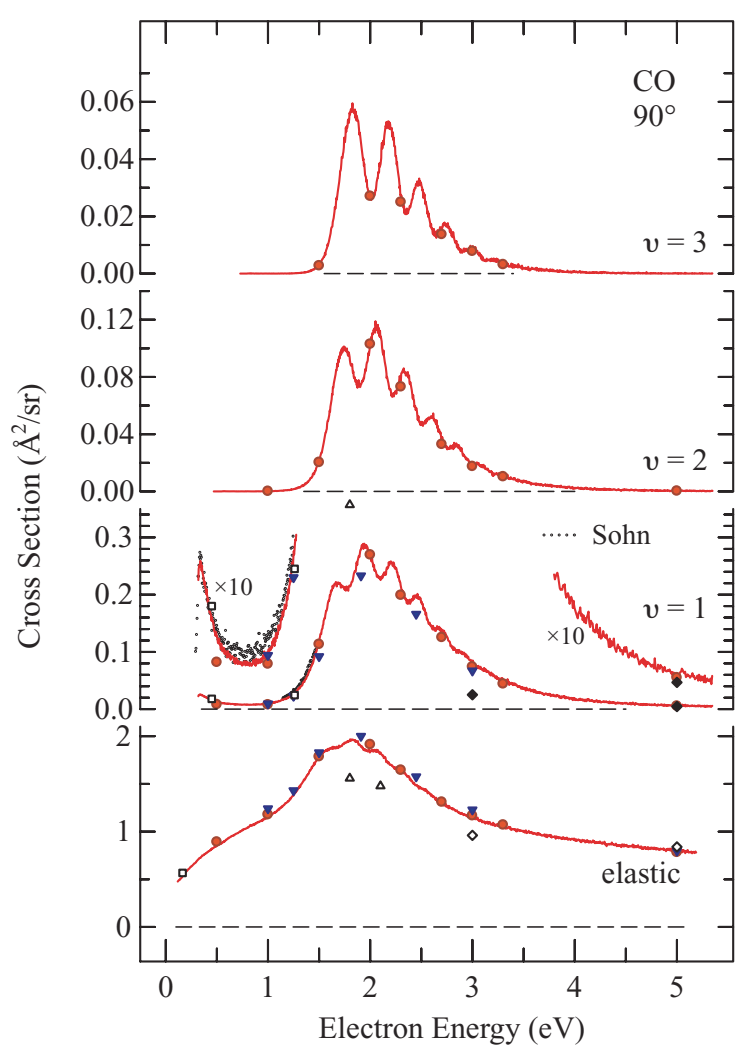

FIG. 5. (Color online) As Fig. 2 but at $90^{\circ}$. Two sets of data of Sohn et al. [12] are shown; dots from their Fig. 3, open squares from their Figs. 1, 4, and 5.

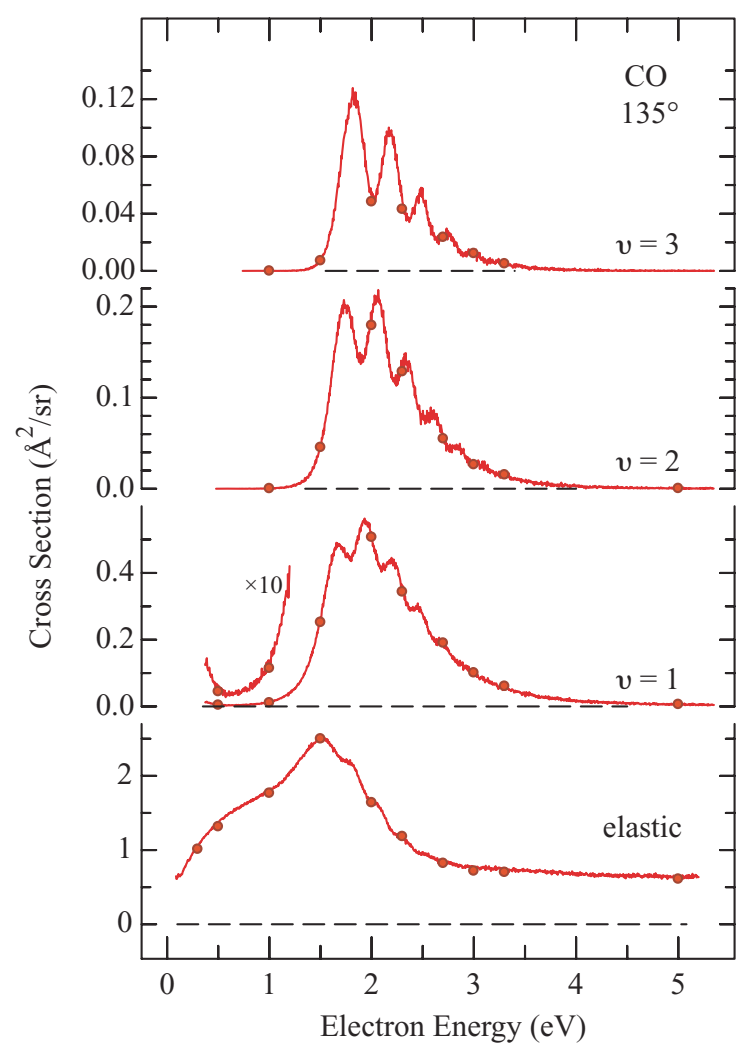

FIG. 6. (Color online) As Fig. 2 but at $135^{\circ}$. 


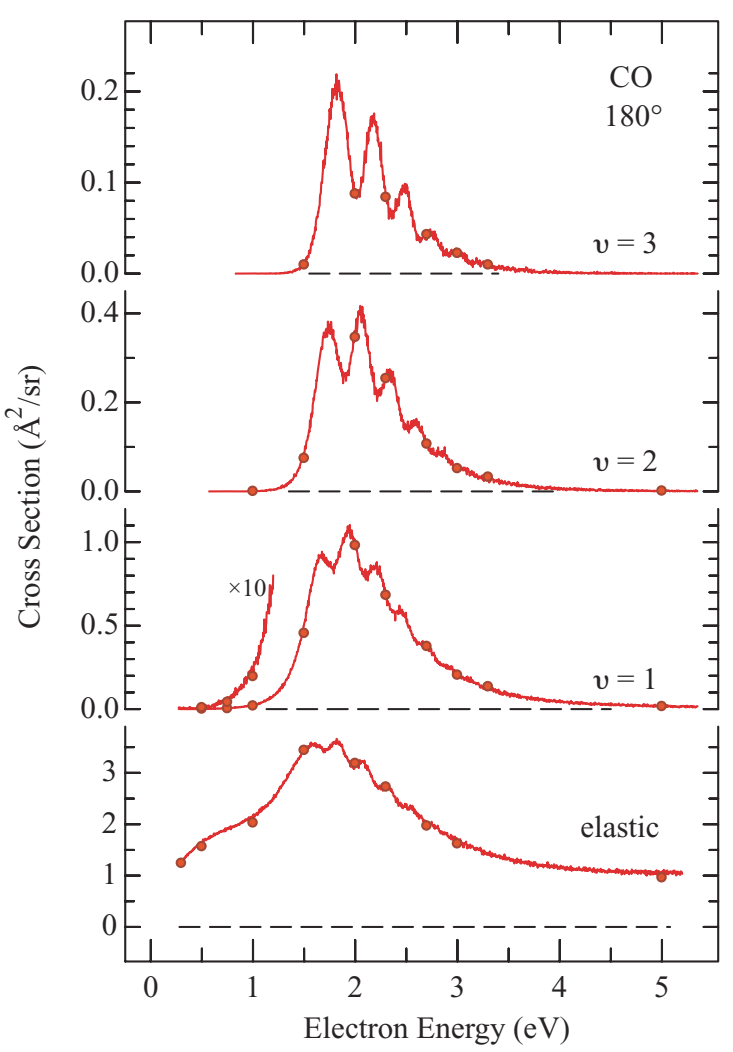

FIG. 7. (Color online) As Fig. 2 but at $180^{\circ}$.

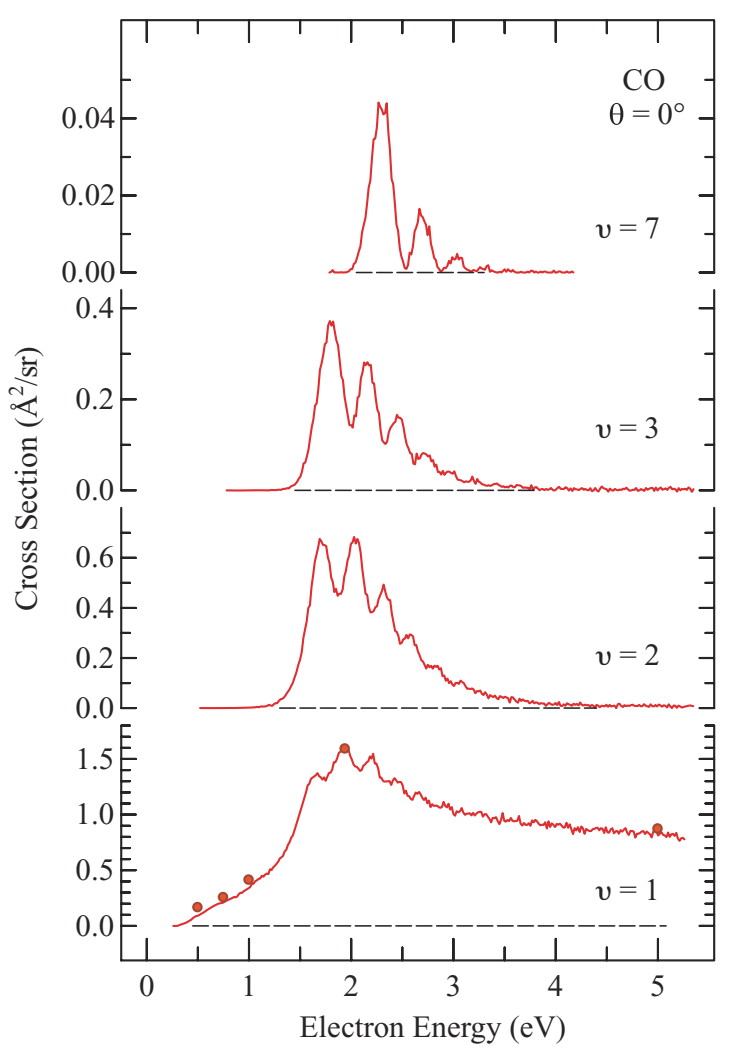

FIG. 8. (Color online) Cross sections for exciting selected vibrational levels at $0^{\circ}$.

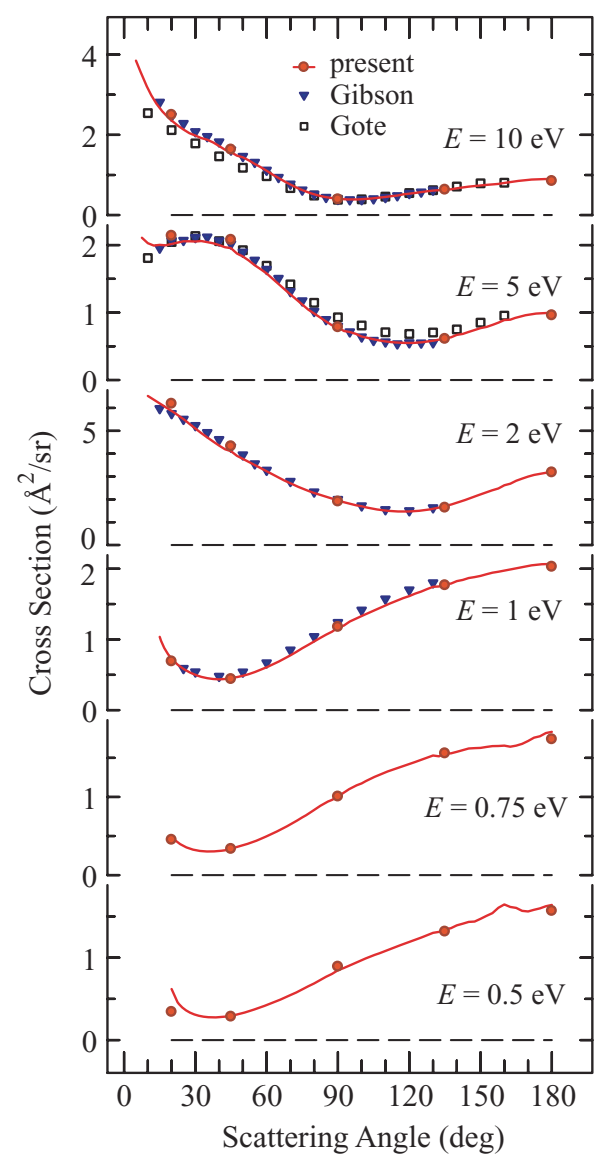

FIG. 9. (Color online) Angular distributions of the elastically scattered electrons. Solid line is the magnetically scanned data with an increment of $2.5^{\circ}$, circles the individual absolute measurements to which the red curves were normalized. The data of Gibson et al. [13] and Gote and Ehrhardt [14] are shown for comparison.

at $0^{\circ}, 20^{\circ}$, and $180^{\circ}$ were recorded with the aid of the magnetic angle changer and the analyzer positioned at $45^{\circ}$ and $135^{\circ}$.

The elastic cross sections agree very well with the measurements of Gibson et al. [13]. The agreement is also very good for $v=1$ excitation below the resonance region at $90^{\circ}$ in Fig. 5. The present $v=1$ cross sections are generally slightly higher than those of Gibson et al. in the resonance region, however. The cross sections measured at very low energies by Sohn et al. [12], and shown as open squares, agree very well with the present data at $90^{\circ}$ in Fig. 5 but less well at $20^{\circ}$ and $45^{\circ}$. The shape of the $v=1$ cross section below the resonance region agrees well with that measured by Sohn et al. [12] at $90^{\circ}$ (shown as dots in Fig. 5). Both experiments show a weak threshold peak. Interestingly, the threshold peak is absent in the forward direction, both in the present $20^{\circ} v=1$ spectrum in Fig. 2 and in the at $12.5^{\circ}$ spectrum of Sohn et al. [12] (not shown here).

The data of Jung et al. [11], shown as upward pointing open triangles in Figs. 2, 4, and 5, is generally lower than the present results for the elastic cross section and higher for the $v=1$ cross section. There is excellent agreement between the present measurement and the cross section calculated for $v=1$ excitation in the resonance region by 


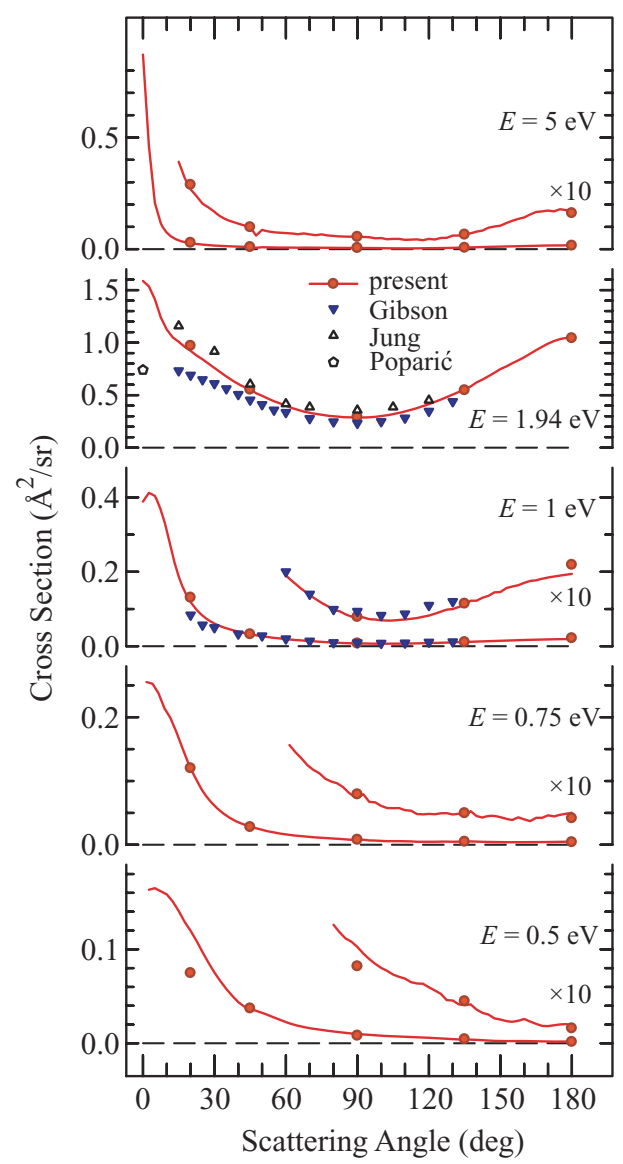

FIG. 10. (Color online) Angular distributions of electrons having excited the $v=1$ level. The data of Gibson et al. [13], Jung et al. [11], and Poparić et al. [16] are shown for comparison.

Gibson et al. [13] (Fig. 2). An illustrative comparison with the 1989 data [15] from the magnetically collimated (trochoidal) spectrometer is given for $v=7$ in Fig. 3. It was normalized to the present data at the peak of the $20^{\circ}, v=1$ spectrum

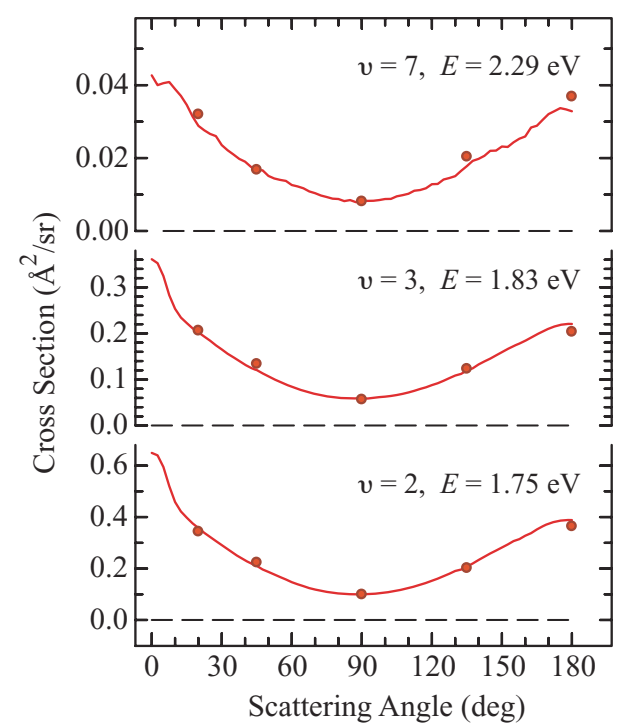

FIG. 11. (Color online) Angular distributions of electrons having excited the $v=2,3$, and 7 levels.
(Fig. 2). It is consistent with the present data, except for a small difference in energy scale calibration, and the fact that the valleys in the present cross section are deeper as a consequence of better resolution. The vibrationally inelastic cross sections are dominated by the ${ }^{2} \Pi$ resonance and their shape as a function of electron energy would thus not be expected to depend on scattering angle. Interestingly, the present data does reveal a weak dependence of the $v=1$ cross section. It is best apparent when the relative depths of the first two valleys in the $v=1$ cross sections, at 1.8 and $2.1 \mathrm{eV}$, are regarded at various scattering angles. At $90^{\circ}$ (Fig. 5), the first valley is deeper than the second; at other angles (apart from $0^{\circ}$ ) it is the other way around.

There is an excellent agreement between the present elastic cross sections and those of Gibson et al. [13] in the angular distributions shown in Fig. 9. The agreement with the data of Gote and Ehrhardt [14], available at 5 and $10 \mathrm{eV}$, is also very good.

The present $v=1$ cross sections, shown in Fig. 10, agree well with those of Gibson et al. below the resonance region but are slightly higher in the resonance region, as already noted above in connection with the figures showing the cross section as a function of incident electron energy. The difference becomes larger below about $30^{\circ}$ at $1.94 \mathrm{eV}$. The data of Jung et al. [11] (open triangles in Fig. 10) agrees very well with the present data in shape, but are slightly higher in magnitude.

The angular distribution in the resonance region, at $1.94 \mathrm{eV}$ in Fig. 10, is nearly symmetric around $90^{\circ}$, with the exception of a peak at $0^{\circ}$. This peak is very similar in shape and height to that observed just below the resonance region, at $1 \mathrm{eV}$ in Fig. 10. This suggests that it is due to an excitation mechanism other than by the ${ }^{2} \Pi$ resonance. This conclusion is supported by the fact that the shape of the cross section recorded as a function of energy at $0^{\circ}$, Fig. 8 , differs somewhat from those recorded at higher angles (Figs. 2, 4, 5, 6, and 7).

Poparic et al. [17] measured the ratio of the forward and backward cross sections in the resonance region to be $1.00 \pm$ 0.06 , in an apparent contradiction to the data in Fig. 10. The two measurements are probably not contradictory; however, the difference could be a consequence of the somewhat peculiar angular response function of the magnetically collimated spectrometers such as used by Poparić et al. Two properties of this type of spectrometers are relevant here [38]:

(a) The acceptance angle is large at low energies, $2 \mathrm{eV}$ scattered electrons were collected up to $\theta \sim 15^{\circ}$ for the spectrometer of Ref. [38].

(b) The spectrometer collects signal for all azimuthal angles $\varphi$. This has the consequence that, although the spectrometer is nominally pointed in the $\theta=0^{\circ}$ direction, its sensitivity at $\theta=0^{\circ}$ is quite low and initially increases with increasing $\theta$. For the spectrometer of Ref. [38] and $2 \mathrm{eV}$ scattered electrons the acceptance scattering angle extends up to $\theta \sim 15^{\circ}$ and the sensitivity peaks at $\theta \sim 8^{\circ}$. This has the consequence that a magnetically collimated spectrometer is relatively insensitive to a narrow peak at $\theta=0^{\circ}$. The present spectrometer has an angular resolution of about $5^{\circ}$ [39], full angle, FWHM, at $2 \mathrm{eV}$, and with an analyzer pass energy of $3 \mathrm{eV}$ used in this work. The real width of the forward peak is thus narrower than it appears to be in Fig. 10. 


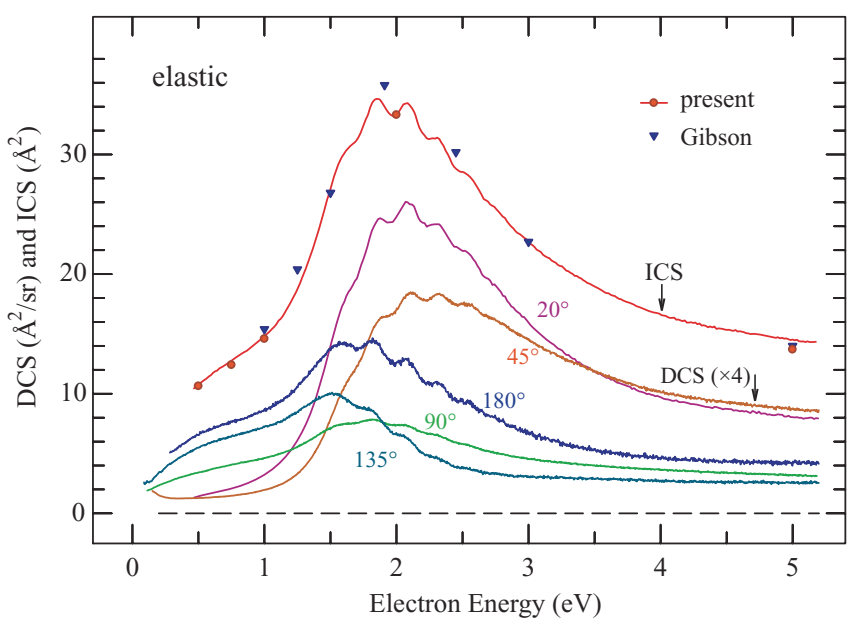

FIG. 12. (Color online) Integral elastic cross section (ICS) obtained by integration under the angular distributions in Fig. 9 are shown as red circles. The top (red) curve shows the shape of the ICS, obtained as a weighted sum of the differential cross sections, shown for comparison below the ICS ( $4 \times$ vertically expanded). The data of Gibson et al. [13] are shown as blue triangles.

The angular distributions for the representative higher levels, $v=2,3$, and 7, shown in Fig. 11 have nearly the same shape, except that the $0^{\circ}$ peak appears to be missing for $v=7$. The shapes of the present elastic and inelastic angular distributions agree well with those of Ehrhardt et al. [8] (not shown here), who measured them for up to $v=6$ at a number of energies in the $20^{\circ}-110^{\circ}$ range.

\section{B. Integral cross sections}

The angular distributions in Figs. 9-11 were multiplied by $2 \pi \sin \theta$ and integrated to obtain integral cross sections. The elastic cross sections were visually extrapolated to $0^{\circ}$ before integrating. The resulting integral cross sections are listed in Table I and shown as red circles in Fig. 12. Momentum-transfer cross sections were obtained in a similar way with an additional $(1-\cos \theta)$ factor and are also listed in Table I.

The integral cross section as a continuous function of energy was then obtained as a weighted superposition of the differential cross sections as indicated in Fig. 12. The entire angular range was divided into five sections with markers at $30^{\circ}, 65^{\circ}, 115^{\circ}, 160^{\circ}$, and the weights were set to the integral of $2 \pi \sin \theta$ between the markers. Although only 5 different angles were taken, the resulting curve is consistent with the 7 discrete values obtained rigorously by integration of angular distributions. This implies that the weighted sum, red curve in Fig. 12, is a good approximation of the integral cross sections. It is in excellent agreement with the integral cross sections of Gibson et al. [13].

TABLE I. Integral (ICS) and momentum transfer (MTCS) elastic cross sections, in $\AA^{2}, \pm 15 \%$ (2 standard deviations).

\begin{tabular}{lcccccr}
\hline \hline Energy (eV) & 0.5 & 0.75 & 1.0 & 2.0 & 5.0 & 10.0 \\
\hline ICS & 10.7 & 12.4 & 14.6 & 33.2 & 13.7 & 11.2 \\
MTCS & 13.5 & 15.7 & 18.1 & 26.2 & 10.4 & 8.1 \\
\hline \hline
\end{tabular}

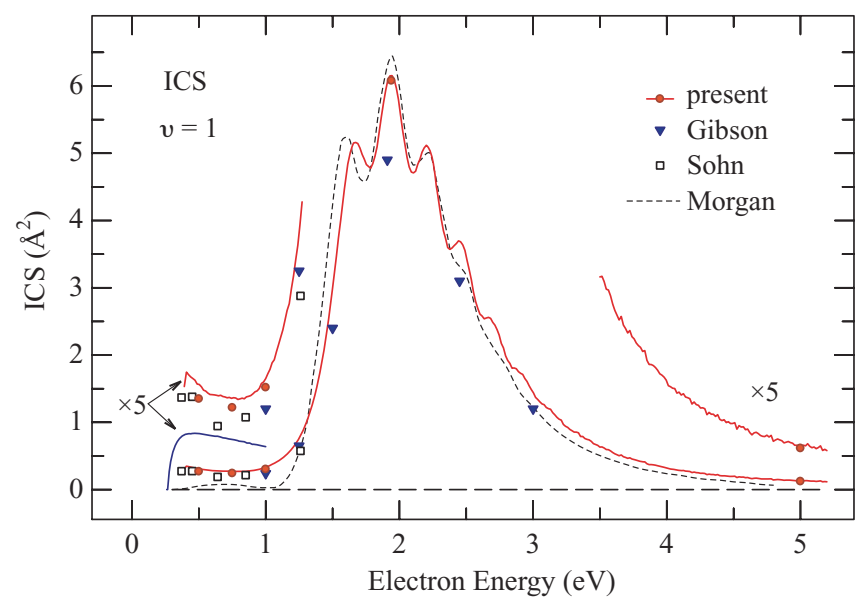

FIG. 13. (Color online) As Fig. 12 but for excitation of $v=1$. Also shown is the data of Gibson et al. [13], Sohn et al. [12], Hake and Phelps [18] (from drift velocity measurements, shown $5 \times$ vertically expanded), and Morgan [26] (theory).

The same procedure was used to obtain integral cross sections for final $v=1,2,3$, and 7 , both by integrating under the angular distributions to obtain integral cross sections at discrete energies and by making a weighted sum of the excitation functions to obtain the ICS as a continuous function of energy. An additional marker was put at $5^{\circ}$ and the spectra recorded at $0^{\circ}$ were included in the sum. A good agreement between the values obtained by integration of angular distributions and values obtained by superposition of differential cross sections was found (Figs. 13-14). Having thus validated the superposition procedure, integral cross sections for $v=4$, 5 , and 6 were obtained by the same superposition. Exactly the same procedure could not be used for $v=8-11$ because differential cross sections were available only at $45^{\circ}, 90^{\circ}$, and $135^{\circ}$. Tests on the lower $v^{\prime}$ s have shown that a superposition of only three spectra is also acceptable, however. The integral

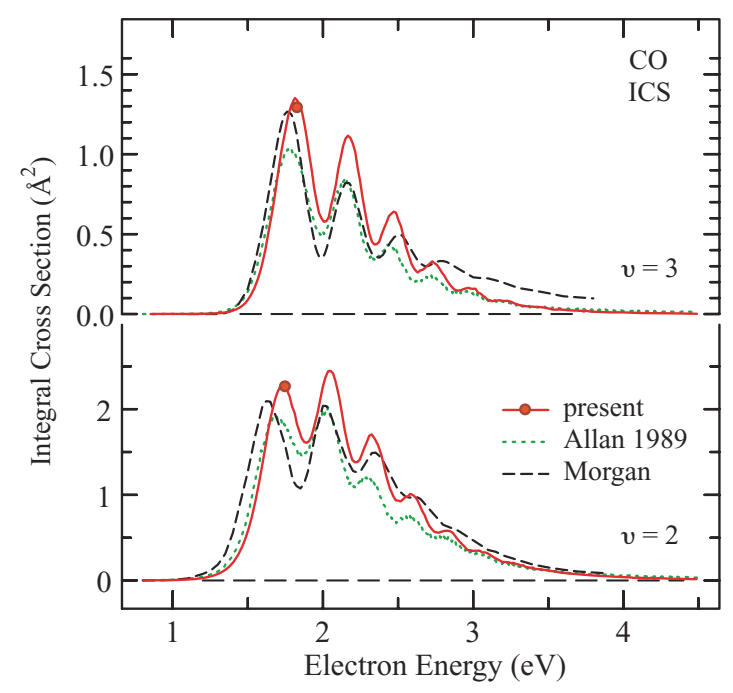

FIG. 14. (Color online) Integral cross sections for excitation of the $v=2$ and 3 levels. The theoretical results of Morgan [26] are shown as dashed line. The early relative experimental data of Allan [15], normalized to the present data at the peak of the $v=1$ cross section, is shown dotted. 
cross sections for $v=8-11$ were consequently obtained as a superposition of the $45^{\circ}, 90^{\circ}$, and $135^{\circ}$ spectra. This amounts to using an assumption of a constant shape of the angular distributions for all $v^{\prime}$ s in the resonant region, an assumption which has been verified up to $v=7$ and is very likely valid for up to $v=11$.

The $v=1$ integral cross sections in Fig. 13 are somewhat higher than those of Gibson et al. [13]. Although the difference is relatively modest, $25 \%$ at $1.94 \mathrm{eV}$, it is much larger than the differences of the elastic values in Fig. 12 and must be considered to be significant. It reflects the difference found already for the differential cross sections. It means that the present ratio of the elastic and $v=1$ peaks in the energy loss spectrum differs from that of Gibson et al. In part it could be a consequence of the fact that the present data was corrected for the instrumental response function, whereas the response function was assumed to be constant by Gibson et al. This effect is, however, unlikely to explain the entire difference between the data, because the energy loss (266 meV) involved in the excitation of the $v=1$ level is relatively small compared to the incident energy around $2 \mathrm{eV}$. Another aspect which could play a role is that integrals under the energy-loss bands were used in the present work. Since the $v=1$ peaks in the energy loss spectra are, because of larger degree of rotational excitation, broader than the elastic peaks, relying on peak heights will underestimate the inelastic cross sections. But this effect should be weak in the work of Gibson et al. because the resolution $(50 \mathrm{meV})$ they used is wide enough to cause an implicit integration under the band. The difference thus remains partly unexplained.

In the resonance region there is an excellent agreement with the calculated cross section of Morgan [26], both in terms of shape and in terms of absolute value. Below the resonance region, the present cross section is in reasonable agreement with that of Sohn et al. [12]. It is, however, nearly twice as high as the cross section derived from transport phenomena by Hake and Phelps [18]. This discrepancy between swarm and beam data was already noted by Schulz [4], and Sohn et al. [12].

Two more inelastic integral cross sections are shown in Fig. 14. The agreement with the theory of Morgan [26] is quite good. The agreement with the old data of the author is acceptable in view of the fact that substantially different type of instrument was used. The old data were only relative and were normalized here to the peak of the $v=1$ cross section. Figure 14 thus indicates a difference between the $(v=1) /(v=2)$ cross-section ratio in the two experiments. This is not entirely unexpected-it is relatively difficult to measure the $v=1$ signal in the magnetically collimated spectrometer. It is not trivial to separate it from the dramatically

TABLE II. Peak elastic and vibrational excitation integral cross sections, in $\AA^{2}, \pm 20 \%$ (2 standard deviations).

\begin{tabular}{lcccccc}
\hline \hline Final $v$ & 0 & 1 & 2 & 3 & 4 & 5 \\
\hline Energy (eV) & 1.86 & 1.94 & 2.05 & 1.82 & 1.94 & 2.04 \\
Peak ICS & 34.7 & 6.13 & 2.43 & 1.34 & 0.85 & 0.53 \\
Final $v$ & 6 & 7 & 8 & 9 & 10 & 11 \\
\hline Energy (eV) & 2.16 & 2.29 & 2.42 & 2.57 & 2.74 & 2.92 \\
Peak ICS & 0.313 & 0.180 & 0.095 & 0.036 & 0.0140 & 0.0044 \\
\hline \hline
\end{tabular}

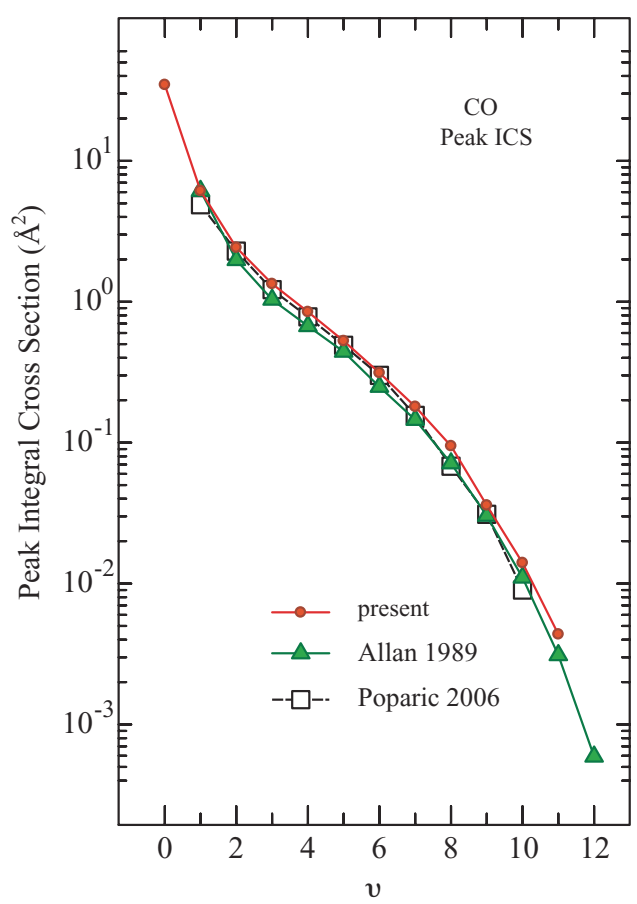

FIG. 15. (Color online) Integral cross sections at energies where they peak, as a function of the final vibrational quantum $v$. The results of Poparic et al. [16] (which they normalized to the $v=1$ ICS of Gibson et al. [13]) and of Allan [15] (normalized to the present $v=1 \mathrm{ICS}$ ) are shown for comparison.

stronger signal of the unscattered electrons and this could possibly affect the $v=1$ signal intensity.

The peak cross sections are given in Table II. The relative magnitudes can be conveniently compared in Fig. 15. It confirms that the main difference between the 1989 and the present data is in the $(v=1) /(v=2)$ ratio, which is larger for the old data. The two curves are essentially parallel for higher $v$. A similar statement can be made with respect to the data of Poparic et al. [16], except that the $(v=1) /(v=2)$ ratio is smaller for their data. The entire set of the present integral cross sections is shown in Fig. 16.

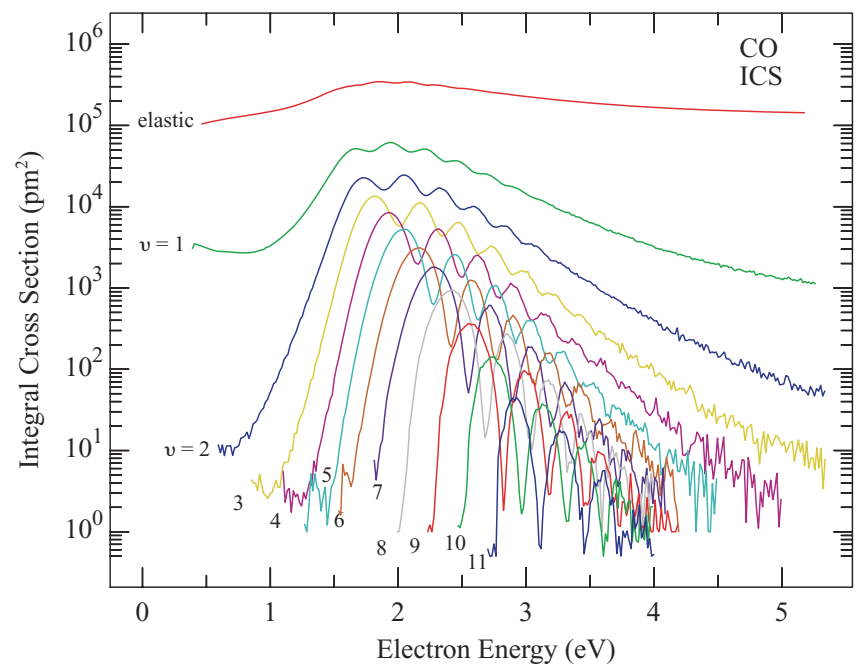

FIG. 16. (Color online) Integral cross sections. 


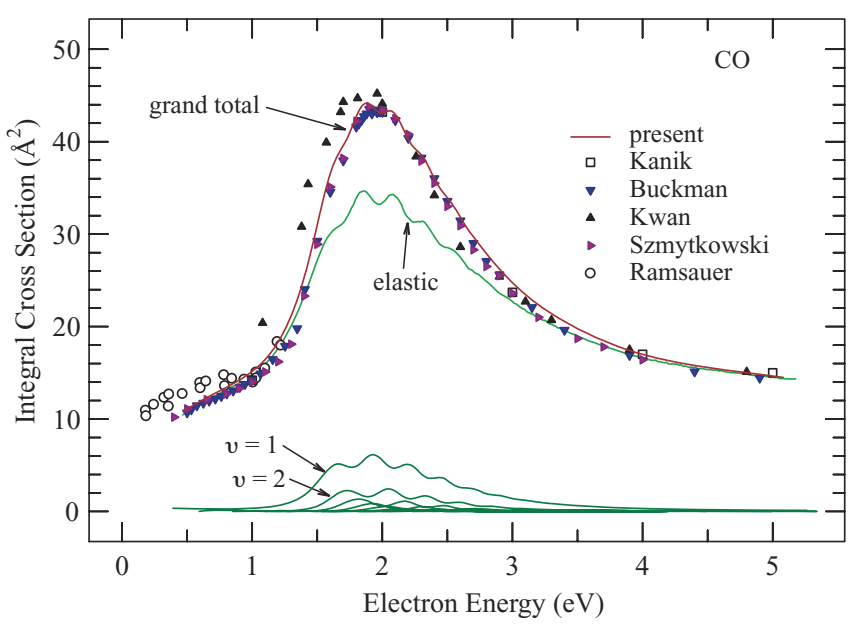

FIG. 17. (Color online) Grand total cross section. The results of Ramsauer and Kollath [20], Buckman and Lohmann [21], Kwan et al. [22], Szmytkowski et al. [23], as well as the "recommended" data of Kanik et al. [24] are shown for comparison. The present elastic and vibrational excitation integral cross sections are also shown.

The sum of the present elastic and inelastic integral cross sections is tested against the grand total cross sections in Fig. 17. This test is important because the grand-total cross sections were measured in transmission-type experiments, which do not have many of the problems encountered in the measurement of partial differential cross sections, like correction for instrumental response function, or use of the relative flow method. The result is very satisfactory, as the present sum agrees very well with the grand total cross sections of Szmytkowski et al. [23] and of Buckman and Lohmann [21]. The experiment of Kwan et al. [22], optimized for positron scattering, yielded cross sections which are larger at energies below $2 \mathrm{eV}$. Part of the problem could be a slight error in energy scale calibration - the agreement would improve if their data were shifted to higher energies by about $0.15 \mathrm{eV}$. The accuracy, and the low-energy capacity, of the 1930 data of Ramsauer and Kollath [20] is remarkable in view of the simplicity of the equipment available at the time.

\section{CONCLUSIONS}

The present measurements improve on the existing data by increasing the angular range-improving the integration leading to the integral cross sections, by carefully determining the instrumental response function on helium and correcting for it, and by integrating under the energy loss bands and thus taking into account varying degree of rotational excitation. Vibrational excitation cross sections were measured up to $v=11$. The sum of the present elastic and all inelastic integral cross sections agrees very well with the available transmission measurements of the grand total cross section. The transmission measurements do not yield information on the final states or the angular distributions, but are a priori more reliable because they do not depend on the corrections for the response functions and do not require the relative flow method. The agreement of the sum of the present data with the transmission measurements thus represents an important validation of the present procedures of correcting for the response function and the relative flow method.

The present elastic differential and integral cross sections are in an excellent agreement with the best available absolute measurement, that of Gibson et al. [13], but the present $v=1$ inelastic integral cross section is about $25 \%$ higher. This could have consequences for simulations of cometary and planetary atmospheres. The discrepancy between cross sections derived from electron transport properties and from beam experiments below the ${ }^{2} \Pi$ resonance region is confirmed.

The detailed shapes of the cross sections, both as a function of energy and of scattering angle, provide reference data for further improvement of theory, both within the ${ }^{2} \Pi$ resonance and below. Two interesting details in this respect are the slightly different shape of the $v=1$ cross section, plotted against electron energy, at $90^{\circ}$ as compared to other angles, and the observation, permitted by the higher resolution of the present experiment, that the valleys between the boomerang structures drop to nearly zero for $v \geqslant 7$.

\section{ACKNOWLEDGMENTS}

This research is part of Project No. 200020-113599/1 of the Swiss National Science Foundation.
[1] L. Campbell and M. J. Brunger, Geophys. Res. Lett. 36, L03101 (2009).

[2] L. Campbell and M. J. Brunger, Phys. Scr. 80, 058101 (2009).

[3] L. Campbell and M. J. Brunger, PMC Phys. B 1, 3 (2008).

[4] G. J. Schulz, Rev. Mod. Phys. 45, 423 (1973).

[5] M. J. Brunger and S. J. Buckman, Phys. Rep. 357, 215 (2002).

[6] A. Zecca, G. P. Karwasz, and R. S. Brusa, Riv. Nuovo Cimento 19, 1 (1996).

[7] G. J. Schulz, Phys. Rev. 135, A988 (1964).

[8] H. Ehrhardt, L. Langhans, F. Linder, and H. S. Taylor, Phys. Rev. 173, 222 (1968).

[9] H. Tanaka, S. K. Srivastava, and A. Chutjian, J. Chem. Phys. 69 , 5329 (1978).

[10] A. Chutjian and H. Tanaka, J. Phys. B: At. Mol. Phys. 13, 1901 (1980).
[11] K. Jung, T. Antoni, R. Müller, K.-H. Kochem, and H. Ehrhardt, J. Phys. B: At. Mol. Phys. 15, 3535 (1982).

[12] W. Sohn, K.-H. Kochem, K. Jung, H. Ehrhardt, and E. S. Chang, J. Phys. B: At. Mol. Phys. 18, 2049 (1985).

[13] J. C. Gibson, L. A. Morgan, R. J. Gulley, M. J. Brunger, C. T. Bundschu, and S. J. Buckman, J. Phys. B: At. Mol. Opt. Phys. 29, 3197 (1996).

[14] M. Gote and H. Ehrhardt, J. Phys. B: At. Mol. Opt. Phys. 28, 3957 (1995).

[15] M. Allan, J. Electron Spectrosc. Relat. Phenom. 48, 219 (1989).

[16] G. B. Poparić, D. S. Belić, and M. D. Vićić, Phys. Rev. A 73, 062713 (2006).

[17] G. B. Poparić, S. M. D. Galijaš, and D. S. Belić, Phys. Rev. A 70, 024701 (2004). 
[18] R. D. Hake Jr. and A. V. Phelps, Phys. Rev. 158, 70 (1967).

[19] J. E. Land, J. Appl. Phys. 49, 5716 (1978).

[20] C. Ramsauer and R. Kollath, Ann. Physik (Leipzig) 4, 91 (1930).

[21] S. J. Buckman and B. Lohmann, Phys. Rev. A 34, 1561 (1986).

[22] C. K. Kwan, Y. F. Hsieh, W. E. Kauppila, S. J. Smith, T. S. Stein, M. N. Uddin, and M. S. Dababneh, Phys. Rev. A 27, 1328 (1983).

[23] C. Szmytkowski, K. Maciag, and G. Karwasz, Phys. Scr. 54, 271 (1996).

[24] I. Kanik, S. Trajmar, and J. C. Nickel, J. Geophys. Res. 98, 7447 (1993).

[25] F. H. Read, J. Phys. B (Proc. Phys. Soc.) 1, 893 (1968).

[26] L. A. Morgan, J. Phys. B: At. Mol. Opt. Phys. 24, 4649 (1991).

[27] L. A. Morgan and J. Tennyson, J. Phys. B: At. Mol. Opt. Phys. 26, 2429 (1993).

[28] M. Allan, J. Phys. B: At. Mol. Opt. Phys. 25, 1559 (1992).
[29] M. Allan, J. Phys. B: At. Mol. Opt. Phys. 38, 3655 (2005).

[30] M. Allan, J. Phys. B: At. Mol. Opt. Phys. 40, 3531 (2007).

[31] A. Gopalan, J. Bömmels, S. Götte, A. Landwehr, K. Franz, M. W. Ruf, H. Hotop, and K. Bartschat, Eur. Phys. J. D 22, 17 (2003).

[32] J. C. Nickel, P. W. Zetner, G. Shen, and S. Trajmar, J. Phys. E: Sci. Instrum. 22, 730 (1989).

[33] R. K. Nesbet, Phys. Rev. A 20, 58 (1979).

[34] F. H. Read and J. M. Channing, Rev. Sci. Instrum. 67, 2372 (1996).

[35] M. Zubek, N. Gulley, G. C. King, and F. H. Read, J. Phys. B: At. Mol. Opt. Phys. 29, L239 (1996).

[36] M. Allan, J. Phys. B: At. Mol. Opt. Phys. 28, 5163 (1995).

[37] See supplementary material at http://link.aps.org/supplemental/ 10.1103/PhysRevA.81.042706.

[38] K. R. Asmis and M. Allan, J. Phys. B: At. Mol. Opt. Phys. 30, 1961 (1997).

[39] M. Allan, J. Phys. B: At. Mol. Opt. Phys. 33, L215 (2000). 